Tohoku J. Exp. Med., 2011, 223, 85-90

\title{
Association between Bone Mineral Density and Arterial Stiffness in Hypertensive Patients
}

\author{
Hisashi Masugata, ${ }^{1}$ Shoichi Senda, ${ }^{1}$ Michio Inukai, ${ }^{1}$ Koji Murao, ${ }^{2}$ \\ Naohisa Hosomi, ${ }^{3}$ Yasuyoshi Iwado, ${ }^{4}$ Takahisa Noma, ${ }^{4}$ Masakazu Kohno, ${ }^{4}$ \\ Nobuyuki Miyatake, ${ }^{5}$ Takashi Himoto ${ }^{1}$ and Fuminori Goda ${ }^{1}$

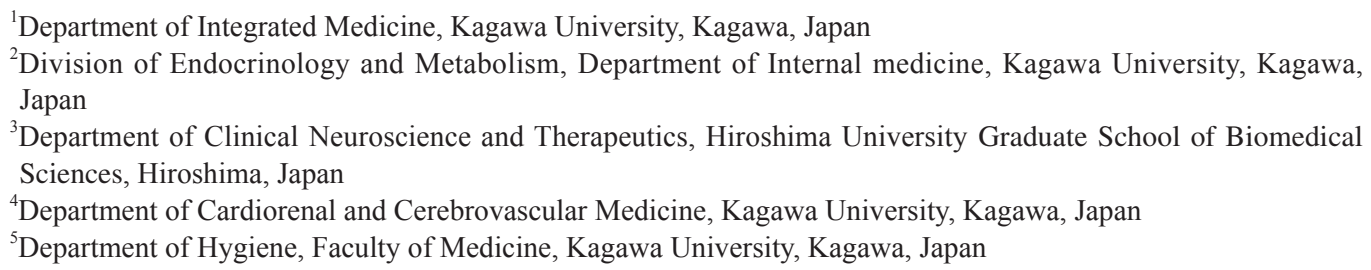

Hypertension and osteoporosis are two common diseases in the elderly population. Recently, reduced bone mineral density has been found in hypertensive patients compared with healthy controls. Reduced bone mineral density is associated with increased arterial stiffness in chronic dialysis patients and healthy postmenopausal women. However, relationships between bone mineral density and arterial stiffness in hypertensive patients have not been fully assessed. We examined the relationships between bone mineral density and both arterial stiffness and nutritional status in 52 hypertensive patients (27 male and 25 female subjects; mean age $71 \pm 8$ years) who had been treated with antihypertensive drugs for at least one year. The bone mineral density of the calcaneus was measured with a quantitative ultrasound measurement device, and the stiffness index was determined as a parameter of the bone mineral density. We measured the cardio-ankle vascular index (CAVI) to assess arterial stiffness and used the serum albumin to assess nutritional status. Increased arterial stiffness as assessed with CAVI is associated with reduced bone mineral density $(r=-0.289, p=0.038)$. However, the correlation between CAVI and bone mineral density is not as strong as the correlation between serum albumin and bone mineral density $(r=0.501, p<0.001)$. In conclusion, nutritional status is an important indicator of bone mineral density in hypertensive patients. Moreover, increased arterial stiffness is associated with reduced bone mineral density in hypertensive patients. Therefore, hypertensive patients with increased arterial stiffness may have a high risk of bone fracture due to osteoporosis.

Keywords: hypertension; bone mineral density; arterial stiffness; cardio-ankle vascular index

Tohoku J. Exp. Med., 2011, 223 (2), 85-90. C 2011 Tohoku University Medical Press

Hypertension and osteoporosis are two common diseases in the elderly population. Reduced bone mineral density has been found to be more common in patients with hypertension than in healthy controls (Cappuccio et al. 1999; Tsuda et al. 2001; Pennisi et al. 2004). Animal and epidemiological studies have suggested that high blood pressure is associated with abnormalities of calcium metabolism, leading to an increase in calcium loss and secondarily, to activation of the parathyroid gland and increased depletion of calcium from the bones, thereby increasing the risk of osteoporosis (Resnick et al. 1983; Imaoka et al. 1991). It is well known that hypertension is a major risk factor of increased arterial stiffness (London et al. 1989; O'Rourke 1990). Therefore, in elderly patients, hyperten- sion may develop into coexisting osteoporosis and arterial stiffening. However, the relationship between osteoporosis and arterial stiffness has not been fully assessed.

It is well known that nutritional status, including serum albumin, dietary protein, and Vitamin $\mathrm{D}$, is a related predictor of bone mineral density (Bawa 2010; Gutiérrez et al. 2010; Jeong et al. 2010; Jesudason and Clifton 2010). Therefore, we hypothesized that arterial stiffness as well as nutritional status may reflect bone mineral density in hypertensive patients. It is well known that increased arterial stiffness detected via an increase in pulse wave velocity predicts an increased risk of cardiovascular events such as stroke and coronary heart disease in patients with essential hypertension (Simon et al. 1998; Weber et al. 2004).

Received November 15, 2010; revision accepted for publication December 23, 2010. doi: 10.1620/tjem.223.85

Correspondence: Hisashi Masugata, M.D., Ph.D., Department of Integrated Medicine, Kagawa University, 1750-1, Miki-cho, Kita-gun, Kagawa 761-0793, Japan.

e-mail: masugata@med.kagawa-u.ac.jp 
Recently, the cardio-ankle vascular index (CAVI), which is a parameter derived from pulse wave velocity, has been used to assess arterial stiffness (Shirai et al. 2006; Miyashita et al. 2010). In the present study, we examined the association between arterial stiffness as assessed by CAVI and bone mineral density by comparing it with the association between nutritional status and bone mineral density in treated hypertensive patients.

\section{Methods}

\section{Subjects}

The study subjects were 52 patients ( 27 male, 25 female; mean age $71 \pm 8$ years, range 50-83 years) who had been diagnosed with hypertension at Kagawa University Hospital and who had regularly visited the outpatient clinic from April, 2009 through August, 2010. Hypertension was defined as systolic blood pressure $\geq 140 \mathrm{mmHg}$ and/or diastolic blood pressure $\geq 90 \mathrm{mmHg}$. Blood pressure was determined using the conventional cuff method. All patients were treated with at least one antihypertensive drug. For at least one year during their enrollment in this study, the antihypertensive drugs did not change for any patients. Patients with a history of heart failure or obvious heart disease were excluded. None of the patients had a history of atherosclerotic cardiovascular disease or stroke. Osteoporosis was assessed by measuring bone mineral density just after measurement of CAVI. Blood samples were taken in the morning after a 12-hour overnight fast. Plasma total cholesterol, triglyceride, and high-density lipoprotein cholesterol (HDL-C), and serum uric acid, creatinine, albumin, and hemoglobin were measured by standard laboratory techniques. The glomerular filtration rate was estimated from the equation for Japanese patients, recently proposed by a working group of the Japanese Chronic Kidney Disease Initiative (Imai et al. 2008) as follows:

$$
\mathrm{eGFR}=194 \times \mathrm{age}^{-0.287} \times(\text { serum creatinine })^{-1.094}(\times 0.739 \text { if female })
$$

Patients with renal dysfunction whose serum creatinine levels were $\geq 1.2 \mathrm{mg} / \mathrm{dL}$ were excluded from the present study. Relationships between bone mineral density and various clinical characteristics, including blood pressure, CAVI, and laboratory data, were analyzed. This protocol was approved by the Ethics Committee of Kagawa University. Informed consent was obtained from all participants.

\section{Assessment of arterial stiffness by measuring CAVI}

CAVI was measured using an automatic vascular screening system (VaseraVS-1000; Fukuda Densi, Tokyo, Japan) with the patient resting in a supine position. Pulse wave velocity (PWV) was obtained by dividing the vascular length by the time it took for the pulse wave to propagate from the aortic valve to the ankle. Systolic blood pressure (SBP) and diastolic blood pressure (DBP) were measured at the brachial artery. The formula used to calculate CAVI was as follows: $\mathrm{CAVI}=a\left[(2 \rho / \Delta \mathrm{P}) \times \ln (\mathrm{SBP} / \mathrm{DBP}) \mathrm{PWV}^{2}\right]+b$, where $\Delta \mathrm{P}$ is SBPDBP, $\rho$ is blood density, and $a$ and $b$ are scale conversion constants to match aortic PWV. The principle underlying CAVI has been described previously (Shirai et al. 2006). The equation is derived from Bramwell-Hill's equation and the stiffness parameter $\beta$, and CAVI was adjusted for blood pressure based on the stiffness parameter $ß$. Therefore, CAVI reflects the stiffness of the aorta, femoral artery and tibial artery as a whole, and theoretically, it is not affected by blood pressure. All these measurements and calculations were performed in VaSera VS-1000. Electrocardiogram (ECG) electrodes were placed on both wrists, a microphone for detecting heart sounds was placed on the sternum, and cuffs were wrapped around both arms and both ankles. After automatic measurements, the values of right and left CAVI were calculated. The averages of the right and left CAVIs were used for analysis.

\section{Measurement of bone mineral density}

The bone mineral density of the right calcaneus (Guglielmi and de Terlizzi 2009; Iida et al. 2010; Mergler et al. 2010) was measured using a quantitative ultrasound measurement device (A-1000 EXPRESS/InSight, GE Healthcare) with the patient resting in a sitting position just after the measurement of CAVI. The stiffness index, which is a parameter of bone mineral density, was determined by the following equation (Yoshimi et al. 2001; Yahata et al. 2002; Zhang et al. 2003; Iida et al. 2010):

$$
\begin{aligned}
& \text { The stiffness index }=0.67 \times(\text { broadband ultrasound attenuation }) \\
& +0.28 \times(\text { speed of sound })-420 .
\end{aligned}
$$

In addition, in order to assess the degree of reduction of bone mineral density, this machine was able to calculate values compared with average values for young people in their 20's. Furthermore, this machine was able to provide values compared with average values for people of the same age, in order to avoid the influences of aging.

\section{Statistical analysis}

Data are expressed as means \pm S.D. Statistical analysis was performed using the SPSS software package (SPSS, Chicago, IL, USA). Linear regression analysis was performed to evaluate the associations between bone mineral density and other variables. Step-wise multiple regression analysis was performed to determine the correlation between bone mineral density and each independent variable. Values of $p<0.05$ were considered to indicate statistical significance.

\section{Results}

Clinical characteristics of subjects

The clinical parameters of the study subjects are summarized in Table 1. The mean systolic brachial blood pressure of all subjects was not particularly high (133 \pm 19 $\mathrm{mmHg}$ ) because all patients' blood pressure was appropriately controlled by medication. The subjects were considerably old ( $71 \pm 8$ years). Their mean value of CAVI $(9.2 \pm$ 1.4) was high, indicating arterial stiffening. The subjects' mean stiffness index for bone mineral density was $71 \pm 18$. This mean index value was $72 \pm 18 \%$ of the mean values for young people in their 20 's, reflecting the effect of aging on the subjects' bone mineral density. However, the mean index value was $96 \pm 23 \%$ of the mean value for the sameage population, indicating that the reduced bone mineral bone density was due to other causes in addition to aging.

\section{Correlation between bone mineral density and clinical vari- ables}

Linear regression analysis was performed to examine the relationships between bone mineral density (the stiff- 
Table 1. Clinical characteristics of the subjects.

\begin{tabular}{|c|c|}
\hline Number (male/female) & $52(27 / 25)$ \\
\hline Age (years) & $71 \pm 8$ \\
\hline Body height (cm) & $159 \pm 8$ \\
\hline Body weight (kg) & $61 \pm 10$ \\
\hline BMI $\left(\mathrm{kg} / \mathrm{m}^{2}\right)$ & $24.2 \pm 3.5$ \\
\hline $\mathrm{SBP}(\mathrm{mmHg})$ & $133 \pm 19$ \\
\hline $\mathrm{DBP}(\mathrm{mmHg})$ & $81 \pm 10$ \\
\hline Pulse pressure (mmHg) & $54 \pm 13$ \\
\hline Heart rate (beats/min) & $64 \pm 10$ \\
\hline Total cholesterol (mg/dL) & $195 \pm 28$ \\
\hline HDL cholesterol (mg/dL) & $53 \pm 12$ \\
\hline Triglyceride (mg/dL) & $134 \pm 82$ \\
\hline Serum creatinine (mg/dL) & $0.77 \pm 0.23$ \\
\hline Serum uric acid (mg/dL) & $5.3 \pm 1.3$ \\
\hline eGFR $\left(\mathrm{mL} / \mathrm{min} / 1.73 \mathrm{~m}^{2}\right)$ & $70.1 \pm 18.8$ \\
\hline Serum albumin $(\mathrm{g} / \mathrm{dL})$ & $4.2 \pm 0.3$ \\
\hline Hemoglobin $(\mathrm{g} / \mathrm{dL})$ & $13.2 \pm 1.4$ \\
\hline Duration of hypertension (years) & $13 \pm 8$ \\
\hline \multicolumn{2}{|l|}{ Drug administration } \\
\hline ARB/ACEI (\%) & 63 \\
\hline $\mathrm{CCB}(\%)$ & 79 \\
\hline$\beta$-blocker (\%) & 25 \\
\hline Diuretic $(\%)$ & 15 \\
\hline CAVI & $9.2 \pm 1.4$ \\
\hline \multicolumn{2}{|l|}{ Bone mineral density } \\
\hline The stiffness index & $71 \pm 18$ \\
\hline $\begin{array}{l}\text { Value compared with young people in their } \\
20 \text { s }(\%)\end{array}$ & $72 \pm 18$ \\
\hline $\begin{array}{l}\text { Value compared with people of the same } \\
\text { age }(\%)\end{array}$ & $96 \pm 23$ \\
\hline
\end{tabular}

BMI, body mass index; SBP, systolic brachial blood pressure; DBP, diastolic brachial blood pressure; HDL, high-density lipoprotein; eGFR, estimated glomerular filtration rate; $\mathrm{ARB}$, angiotensin II receptor blocker; ACEI, angiotensin-converting enzyme inhibitor; $\mathrm{CCB}$, calcium channel blocker; CAVI, cardio-ankle vascular index.

ness index) and clinical variables in all subjects (Table 2). Among the clinical variables, age $(r=-0.446, p=0.001)$, body height ( $r=0.307, p=0.027)$, body weight $(r=0.276$, $p=0.048)$, serum albumin $(r=0.501, p<0.001)$, hemoglo$\operatorname{bin}(r=0.476, p<0.001)$, and CAVI $(r=-0.289, p=0.038)$ showed significant correlations with the stiffness index of bone mineral density. Among the clinical parameters, serum albumin demonstrated the highest correlation with the stiffness index of bone mineral density. Although the correlation between CAVI and bone mineral density was statistically significant, the correlation was not as strong as that between serum albumin and bone mineral density (Fig. $1)$.

\section{Assessment of the factors related to bone mineral density}

Stepwise multiple regression analysis was performed
Table 2. Correlation coefficients of linear regression analysis between bone mineral density (the stiffness index) and other parameters.

\begin{tabular}{lcc}
\hline & $r$ & $p$ value \\
\hline Age & -0.446 & 0.001 \\
Body height & 0.307 & 0.027 \\
Body weight & 0.276 & 0.048 \\
BMI & 0.114 & $\mathrm{NS}$ \\
SBP $(\mathrm{mmHg})$ & -0.031 & $\mathrm{NS}$ \\
DBP $(\mathrm{mmHg})$ & 0.107 & $\mathrm{NS}$ \\
Pulse pressure $(\mathrm{mmHg})$ & 0.001 & $\mathrm{NS}$ \\
Heart rate $($ beats $/ \mathrm{min})$ & 0.088 & $\mathrm{NS}$ \\
Total cholesterol $(\mathrm{mg} / \mathrm{dL})$ & 0.097 & $\mathrm{NS}$ \\
HDL cholesterol $(\mathrm{mg} / \mathrm{dL})$ & -0.151 & $\mathrm{NS}$ \\
Triglyceride $(\mathrm{mg} / \mathrm{dL})$ & 0.222 & $\mathrm{NS}$ \\
Serum creatinine $(\mathrm{mg} / \mathrm{dL})$ & 0.035 & $\mathrm{NS}$ \\
Serum uric acid $(\mathrm{mg} / \mathrm{dL})$ & -0.041 & $\mathrm{NS}$ \\
eGFR (mL/min/1.73 m $\left.{ }^{2}\right)$ & 0.022 & $\mathrm{NS}$ \\
Serum albumin $(\mathrm{g} / \mathrm{dL})$ & 0.501 & $<0.001$ \\
Hemoglobin $(\mathrm{g} / \mathrm{dL})$ & 0.476 & $<0.001$ \\
CAVI & -0.289 & 0.038 \\
\hline
\end{tabular}

BMI, body mass index; SBP, systolic brachial blood pressure; DBP, diastolic brachial blood pressure; HDL, high-density lipoprotein; eGFR, estimated glomerular filtration rate; CAVI, cardio-ankle vascular index.

to identify which clinical parameters were independently associated with the stiffness index of bone mineral density. Stepwise multiple regression analysis was performed for variables showing significant correlations with the stiffness index of bone mineral density in the linear regression analysis in Table 2 . This analysis indicated that serum albumin $(B$ coefficient $=0.361, p=0.009)$ and hemoglobin ( $\beta$ coefficient $=0.321, p=0.019$ ) were independently associated with the stiffness index of bone mineral density (Table 3 ).

\section{Discussion}

The present study compares CAVI and nutritional status data to identify the association between arterial stiffness and bone mineral density in treated hypertensive patients. The data indicate that increased arterial stiffness as assessed by CAVI is associated with reduced bone mineral density. However, the correlation between CAVI and bone mineral density was not as strong as that between serum albumin and bone mineral density.

Although previous studies (Aoki et al. 2009; Benetos et al. 2009; Mikumo et al. 2009; Seo et al. 2009) have shown that reduced bone mineral density is associated with arterial stiffening in chronic dialysis patients and healthy postmenopausal women, there are no data regarding the association between bone mineral density and arterial stiffness in hypertensive patients without overt cardiovascular diseases. Our results indicate an association between bone mineral density and arterial stiffness in hypertensive 

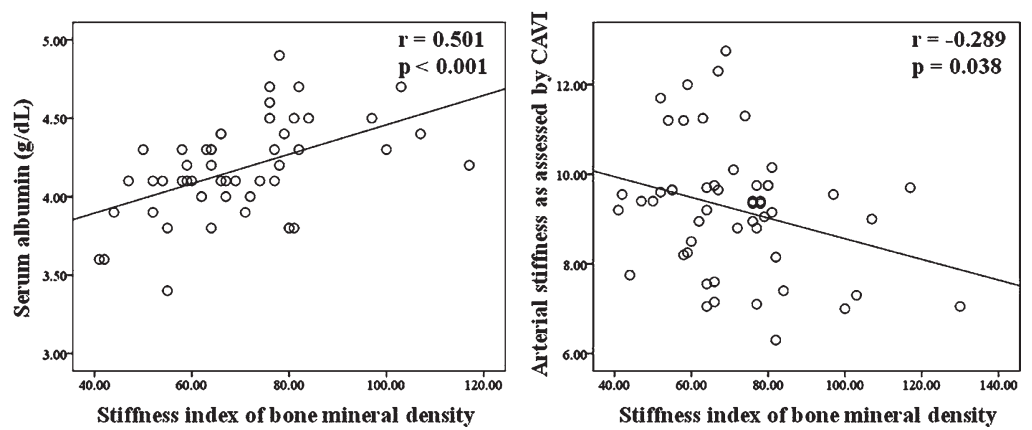

Fig. 1. Relationships between serum albumin, arterial stiffness as assessed by cardio-ankle vascular index (CAVI), and the stiffness index of bone mineral density.

Although the correlation between CAVI and bone mineral density was statistically significant, the correlation was weak compared with that between serum albumin and bone mineral density.

Table 3. Multiple regression analysis of bone mineral density and significantly associated variables.

\begin{tabular}{lccc}
\hline $\begin{array}{c}\text { Independent } \\
\text { variable }\end{array}$ & $\beta$ coefficient & $t$ value & $p$ value \\
\hline Serum albumin & 0.361 & 2.728 & 0.009 \\
Hemoglobin & 0.321 & 2.430 & 0.019 \\
& $F$ ratio $=11.807$ & $r^{2}=0.306$ & \\
\multicolumn{3}{c}{$(p<0.001)$} \\
\hline
\end{tabular}

patients. Our results provide the new finding that nutritional status, including serum albumin and hemoglobin, is a more important determinant of bone mineral density than arterial stiffness in hypertensive patients. Therefore, improvement of nutritional status may be important to prevent bone fractures due to osteoporosis in hypertensive patients. However, our results suggest that increased arterial stiffness is associated with reduced bone mineral density to some degree. Therefore, hypertensive patients with increased arterial stiffness may have a high risk of bone fractures due to osteoporosis. In the present study, arterial stiffness was assessed by CAVI, which is less influenced by blood pressure (Shirai et al. 2006). In addition, the blood pressure of participants was well controlled by medications. Therefore, the blood pressure was not correlated with bone mineral density, whereas CAVI was (Table 2).

It is well known that nutrition status, including serum albumin, dietary protein, and Vitamin $\mathrm{D}$, is a predictor of bone mineral density (Bawa 2010; Gutiérrez et al. 2010; Jeong et al. 2010; Jesudason and Clifton 2010). Our data are in agreement with these previous reports (Jeong et al. 2010; Jesudason and Clifton 2010). Our results suggest that the role of nutrition in bone health is important in treated hypertensive patients as well as chronic dialysis patients or healthy postmenopausal women. However, angiotensin-converting enzyme (ACE) inhibitors have been recently reported to reduce the risk of bone fractures (Lynn et al. 2006; Rejnmark et al. 2006). In addition, a recent experimental study has demonstrated that angiotensin II activates osteoclasts, leading to osteoporosis (Shimizu et al. 2008) and that ACE inhibitors attenuate osteoporosis in spontaneously hypertensive rats (Shimizu et al. 2009). An activated renin-angiotensin system is well known to be a promoting factor of atherosclerosis including arterial stiffening (Shapiro et al. 2008; Sie et al. 2009). The activated renin-angiotensin system in hypertensive patients may have played an important role in the association between reduced bone mineral density and increased arterial stiffness in the subjects of the present study. The participants of the present study included 33 patients with renin-angiotensin system (RAS) inhibitors and 19 patients without RAS inhibitors. The bone mineral density did not differ between patients with RAS inhibitors $(72 \pm 19)$ and patients without RAS inhibitors $(70 \pm 18)$. However, the present study was a cross-sectional study. A follow-up study during antihypertensive treatment may be needed to assess the influence of RAS inhibitors on bone mineral density.

The present study has several limitations. First, we did not measure serum levels of ionized calcium and parathyroid hormone, which might suggest that hypertension is associated with not only increased arterial stiffness but also osteoporosis. Second, we did not assess nutrition habits, including dietary protein and Vitamin $\mathrm{D}$, which may influence bone mineral density. In addition, we did not assess the effects of lifestyle modification such as sodium reduction, which may reduce arterial stiffness, because the present study was a cross-sectional study. Third, we used an ultrasound device to measures bone mineral density in the present study. Although ultrasound measurement is a quick and non-stressful method, bone mineral density is usually by dual-energy X-ray absorptiometry in the clinical settings. However a previous study (Iida et al. 2010) demonstrated that bone mineral density by ultrasound measurement was significantly correlated with bone mineral density on dual-energy X-ray absorptiometry. Finally, it was difficult to determine the difference between genders because the study population of the present study was small. However, the correlation between bone mineral density and CAVI was statistically significant in 27 male patients $(r=$ $-0.542, p=0.004)$, but was insignificant in 25 female patients $(r=-0.131, p=0.531)$ in the present study. A further study containing a large number of male and female 
patients may be needed to elucidate the differences between genders.

In conclusion, nutritional status may be a more important indicator of bone mineral density in hypertensive patients than arterial stiffness. However, increased arterial stiffness is associated with reduced bone mineral density to some degree in hypertensive patients. Therefore, hypertensive patients with increased arterial stiffness may have a high risk of bone fractures due to osteoporosis.

\section{References}

Aoki, A., Kojima, F., Uchida, K., Tanaka, Y. \& Nitta, K. (2009) Associations between vascular calcification, arterial stiffness and bone mineral density in chronic hemodialysis patients. Geriatr. Gerontol. Int., 9, 246-252.

Bawa, S. (2010) The significance of soy protein and soy bioactive compounds in the prophylaxis and treatment of osteoporosis. J. Osteoporos., 891058.

Benetos, A., Zervoudaki, A., Kearney-Schwartz, A., Perret-Guillaume, C., Pascal-Vigneron, V., Lacolley, P., Labat, C. \& Weryha, G. (2009) Effects of lean and fat mass on bone mineral density and arterial stiffness in elderly men. Osteoporos. Int., 20, 1385-1391.

Cappuccio, F.P., Meilahn, E., Zmuda, J.M. \& Cauley, J.A. (1999) High blood pressure and bone-mineral loss in elderly white women: a prospective study. Study of Osteoporotic Fractures Research Group. Lancet, 354, 971-975.

Guglielmi, G. \& de Terlizzi, F. (2009) Quantitative ultrasound in the assessment of osteoporosis. Eur. J. Radiol., 71, 425-431.

Gutiérrez, O.M., Farwell, W.R., Kermah, D. \& Taylor, E.N. (2010) Racial differences in the relationship between vitamin $\mathrm{D}$, bone mineral density, and parathyroid hormone in the National Health and Nutrition Examination Survey. Osteoporos. Int. [Epub ahead of print].

Iida, T., Chikamura, C., Aoi, S., Ikeda, H., Matsuda, Y., Oguri, Y., Ono, Y., Katada, K. \& Ishizaki, F. (2010) A study on the validity of quantitative ultrasonic measurement used the bone mineral density values on dual-energy X-ray absorptiometry in young and in middle-aged or older women. Radiol. Phys. Technol., 3, 113-119.

Imai, E., Matsuo, S., Makino, H., Watanabe, T., Akizawa, T., Nitta, K., Iimuro, S., Ohashi, Y. \& Hishida, A.; CKD-JAC Study Group. (2008) Chronic Kidney Disease Japan Cohort (CKDJAC) study: design and methods. Hypertens. Res., 31, 11011107.

Imaoka, M., Morimoto, S., Kitano, S., Fukuo, F. \& Ogihara, T. (1991) Calcium metabolism in elderly hypertensive patients: possible participation of exaggerated sodium, calcium and phosphate excretion. Clin. Exp. Pharmacol. Physiol., 18, 631-641.

Jeong, J.U., Lee, H.K., Kim, Y.J., Kim, J.S., Kang, S.S. \& Kim, S.B. (2010) Nutritional markers, not markers of bone turnover, are related predictors of bone mineral density in chronic peritoneal dialysis patients. Clin. Nephrol., 74, 336-342.

Jesudason, D. \& Clifton, P. (2011) The interaction between dietary protein and bone health. J. Bone. Miner. Metab., 29, 1-4.

London, G.M., Marchais, S.J. \& Safar, M.E. (1989) Arterial compliance in hypertension. J. Hum. Hypertens., 3 Suppl 1, 53-56.

Lynn, H., Kwok, T., Wong, S.Y., Woo, J. \& Leung, P.C. (2006) Angiotensin converting enzyme inhibitor use is associated with higher bone mineral density in elderly Chinese. Bone, 38, 584-588.

Mergler, S., Löbker, B., Evenhuis, H.M. \& Penning, C. (2010) Feasibility of quantitative ultrasound measurement of the heel bone in people with intellectual disabilities. Res. Dev.
Disabil., 31, 1283-1290.

Mikumo, M., Okano, H., Yoshikata, R., Ishitani, K. \& Ohta, H. (2009) Association between lumbar bone mineral density and vascular stiffness as assessed by pulse wave velocity in postmenopausal women. J. Bone Miner. Metab., 27, 89-94.

Miyashita, Y., Endo, K., Saiki, A., Ban, N., Nagumo, A., Yamaguchi, T., Kawana, H., Nagayama, D., Ohira, M., Oyama, T. \& Shirai, K. (2010) Effect of ezetimibe monotherapy on lipid metabolism and arterial stiffness assessed by cardio-ankle vascular index in type 2 diabetic patients. $J$. Atheroscler. Thromb., 17, 1070-1076.

O'Rourke, M. (1990) Arterial stiffness, systolic blood pressure, and logical treatment of arterial hypertension. Hypertension, 15, 339-347.

Pennisi, P., Signorelli, S.S., Riccobene, S., Celotta, G., Di Pino, L., La Malfa, T. \& Fiore, C.E. (2004) Low bone density and abnormal bone turnover in patients with atherosclerosis of peripheral vessels. Osteoporos. Int., 15, 389-395.

Rejnmark, L., Vestergaard, P. \& Mosekilde, L. (2006) Treatment with beta-blockers, ACE inhibitors, and calcium-channel blockers is associated with a reduced fracture risk: a nationwide case-control study. J. Hypertens., 24, 581-589.

Resnick, L.M., Laragh, J.H., Sealey, J.E. \& Alderman, M.H. (1983) Divalent cations in essential hypertension. Relations between serum ionized calcium, magnesium, and plasma renin activity. N. Engl. J. Med., 309, 888-891.

Seo, S.K., Cho, S., Kim, H.Y., Choi, Y.S., Park, K.H., Cho, D.J. \& Lee, B.S. (2009) Bone mineral density, arterial stiffness, and coronary atherosclerosis in healthy postmenopausal women. Menopause, 16, 937-943.

Shapiro, Y., Boaz, M., Matas, Z., Fux, A. \& Shargorodsky, M. (2008) The association between the renin-angiotensin-aldosterone system and arterial stiffness in young healthy subjects. Clin. Endocrinol. (Oxf.), 68, 510-512.

Shimizu, H., Nakagami, H., Osako, M.K., Hanayama, R., Kunugiza, Y., Kizawa, T., Tomita, T., Yoshikawa, H., Ogihara, T. \& Morishita, R. (2008) Angiotensin II accelerates osteoporosis by activating osteoclasts. FASEB J., 22, 2465-2475.

Shimizu, H., Nakagami, H., Osako, M.K., Nakagami, F., Kunugiza, Y., Tomita, T., Yoshikawa, H., Rakugi, H., Ogihara, T. \& Morishita, R. (2009) Prevention of osteoporosis by angiotensin-converting enzyme inhibitor in spontaneous hypertensive rats. Hypertens. Res., 32, 786-790.

Shirai, K., Utino, J., Otsuka, K. \& Takata, M. (2006) A novel blood pressure-independent arterial wall stiffness parameter; Cardio Ankle Vascular Index (CAVI). J. Atheroscler. Thromb., 13, 101-107.

Sie, M.P., Yazdanpanah, M., Mattace-Raso, F.U., Uitterlinden, A.G., Hofman, A., Hoeks, A.P., Reneman, R.S., Asmar, R., Van Duijn, C.M. \& Witteman, J.C. (2009) Genetic variation in the renin-angiotensin system and arterial stiffness. The Rotterdam Study. Clin. Exp. Hypertens., 31, 389-399.

Simon, A., Megnien, J.L., Gariepy, J. \& Levenson, J. (1998) Early atherosclerosis in human hypertension. Am. J. Hypertens., 11, 882-883.

Tsuda, K., Nishio, I., \& Masuyama, Y. (2001) Bone mineral density in women with essential hypertension. Am. J. Hypertens., 14, 704-707.

Weber, T., Auer, J., O’Rourke, M.F., Kvas, E., Lassnig, E., Berent, R. \& Eber, B. (2004) Arterial stiffness, wave reflections, and the risk of coronary artery disease. Circulation, 109, 184-189.

Yahata, Y., Aoyagi, K., Okano, K., Yoshimi, I., Kusano, Y., Kobayashi, M., Moji, K. \& Takemoto, T. (2002) Metacarpal bone mineral density, body mass index and lifestyle among postmenopausal Japanese women: relationship of body mass index, physical activity, calcium intake, alcohol and smoking to bone mineral density: the Hizen-Oshima study. Tohoku J. Exp. Med., 196, 123-129.

Yoshimi, I., Aoyagi, K., Okano, K., Yahata, Y., Kusano, Y., Moji, 
K., Tahara, Y. \& Takemoto, T. (2001) Stiffness index of the calcaneus measured by quantitative ultrasound and menopause among Japanese women: the Hizen-Oshima Study. Tohoku J. Exp. Med., 195, 93-99.

Zhang, Y., Aoyagi, K., Honda, S., Yahata, Y., Yoshimi, I., Kusano,
Y., Takamura, N., Kobayashi, M., Moji, K. \& Takemoto, T. (2003) Effects of lifestyle factors on stiffness index of calcaneus measured by quantitative ultrasound system among Japanese women aged 40 years and over: the Hizen-Oshima Study. Tohoku J. Exp. Med., 201, 97-107. 